\title{
Computing FGZ Index of Sum Graphs under Strong Product
}

\author{
Zhi-Ba Peng, ${ }^{1}$ Saira Javed, ${ }^{2}$ Muhammad Javaid $\mathbb{D}^{2},{ }^{2}$ and Jia-Bao Liu $\mathbb{D}^{1}$ \\ ${ }^{1}$ School of Mathematics and Physics, Anhui Jianzhu University, Hefei 230601, China \\ ${ }^{2}$ Department of Mathematics, School of Science, University of Management and Technology, Lahore 54770, Pakistan
}

Correspondence should be addressed to Muhammad Javaid; javaidmath@gmail.com

Received 2 January 2021; Revised 10 February 2021; Accepted 11 March 2021; Published 30 March 2021

Academic Editor: Ahmet Sinan Cevik

Copyright (c) 2021 Zhi-Ba Peng et al. This is an open access article distributed under the Creative Commons Attribution License, which permits unrestricted use, distribution, and reproduction in any medium, provided the original work is properly cited.

Topological index (TI) is a function that assigns a numeric value to a (molecular) graph that predicts its various physical and structural properties. In this paper, we study the sum graphs (S-sum, R-sum, Q-sum and T-sum) using the subdivision related operations and strong product of graphs which create hexagonal chains isomorphic to many chemical compounds. Mainly, the exact values of first general Zagreb index (FGZI) for four sum graphs are obtained. At the end, FGZI of the two particular families of sum graphs are also computed as applications of the main results. Moreover, the dominating role of the FGZI among these sum graphs is also shown using the numerical values and their graphical presentations.

\section{Introduction}

Let $G=(V(G), E(G))$ be a (molecular) graph with $V(G)$ and $E(G)$ as sets of vertex and edge respectively. The degree of a vertex $v \varepsilon V(G)$ is the number of edges which are incident to $v$. A topological index (TI) is a function that assigns a numeric value to the under study (molecular) graph, see [1-3]. TIs are used to predict the various chemical and structural properties such as octane isomers including entropy, acentric factor, density, total surface area, molar volume, boiling point, capacity of heat at temperature and pressure, enthalpy of formation, connectivity of compounds and octanol water partition, see [4]. These are also used to study the quantitative structure property and activity relationships which are very important in the subject of cheminformatics, see [5].

Wiener see [6] defined a distance-based TI to compute the boiling point of paraffin. Gutman and Trinajstic see [7] investigated total $\pi$-electron energy of the molecule graphs using a degree-based TI called by first Zagreb index nowadays. Later on, various Zagreb indices (hyper, forgotten, multiplicative and augmented) are defined to predict the different physicochemical properties chirality, heterosystems, complexity, branching and ZE-isomerism of the (molecular) graphs see [8]. Li and Zheng defined the first general Zagreb index (FGZI) and studied its different properties see [9].

The operations of graphs (addition, deletion, complement, product, union and intersection) also play a very important role for the construction of new graphs, see [10]. In particular, for a graph $G$ Yan et al. [11] introduced the five new graphs $S(G), R(G), L(G), Q(G)$ and $T(G)$ are defined with the help of the operations of subdivision $S$, semitotal point $R$, Line graph $L$, semitotal edge $Q$ and total vertex and edge $T$ respectively. Also, Wiener index of these $\phi(G)$ graphs is computed, where $\Phi \in\{S, R, L, Q, T\}$. Eliasi and Taeri [12] constructed the $\Phi$-sum graphs $G_{\Phi}+H$ by the Cartesian product of the graphs $\Phi(G)$ and $H$, where $\Phi(G)$ is obtained after applying the $\Phi$ on $G$ for $\Phi \in\{S, R, Q, T\}$. Moreover, they computed the Wiener indices of the $\Phi$-sums graphs $G_{S}+H, G_{R}+H, G_{Q}+H$ and $G_{T}+H$. Deng et al. [13] computed the first and second Zagreb indices of four operations on graphs using the concept of Cartesian product. Akhter and Imran [14] computed the forgotten topological index of four operations on graphs under Cartesian product. Liu et al. [15] computed the FGZI of $\Phi$-sum graphs under the operation of Cartesian product. Sarala et al. [16] computed the $F$-index of $\Phi$-sum graphs under the operation of strong product. For further study, we refer to [17-29].

In this paper, we extend this study and compute FGZI of the $\Phi$-sums graphs $\left(G_{\Phi} \otimes H\right)$ under the operation of strong product of $\Phi(G)$ and $H$ in term of FGZI of its factor graphs $G$ and $H$, where $\Phi \in\{S, R, Q, T\}, G$ and $H$ are any two connected graphs. The obtained results are general extension of the results of Deng et al. [13], Akhter and Imran [14], Liu et al. [15] and Sarala et al. [16] works. Forthcoming sections 
are arranged as: Section 2 includes the basic formulae and results, Section 3 covers the main results and Section 4 includes the conclusion.

\section{Preliminaries}

An atom is presented by a vertex and bonding between atom is showed by edge in the molecular graphs. The first and second Zagreb indices $M_{1}(G)$ and $M_{2}(G)$ of $G$ are defined as follows $[5,7,22]$ :

$$
\begin{aligned}
& M_{1}(G)=\sum_{v \in V(G)}\left[d_{G}(v)\right]^{2}=\sum_{u v \in E(G)}\left[d_{G}(u)+d_{G}(v)\right], \\
& M_{2}(G)=\sum_{u v \in E(G)}\left[d_{G}(u) d_{G}(v)\right] .
\end{aligned}
$$

For any real number $\alpha$ FGZ index and general Randic index are defined as

$$
\begin{aligned}
M^{\alpha} & =\sum_{v \in V(G)} d_{G}^{\alpha}(v)=\sum_{u v \in E(G)}\left[d_{G}^{\alpha-1}(u)+d_{G}^{\alpha-1}(v)\right], \\
R_{\alpha}(G) & =\sum_{u v \in E(G)}\left[d_{G}(u) d_{G}(v)\right]^{\alpha} .
\end{aligned}
$$

For more details of aforesaid TIs, see [30, 31]. The following graphs are defined with the help of the subdivision related operations $(S, R, Q$ and $T)$, see [16].

(i) By inserting a new vertex in each $u v \in E(G), S(G)$ is obtained.

(ii) By joining the end (original) vertices of the edges being incidence on each new vertex in $S(G), R(G)$ is obtained.

(iii) By joining the new vertices which have common adjacent (original) vertices in $S(G), Q(G)$ is obtained.

(iv) By operating both $R(G)$ and $Q(G)$ on $S(G), T(G)$ is obtained. For more explanation, see Figure 1.

In 1960 Sabidussi introduced the strong product for any two graphs $G \circ H$ has vertex set Cartesian product $V(G) \times$ $V(H)$ such that $\left(s_{1}, t_{1}\right)$ and $\left(s_{2}, t_{2}\right)$ will be adjacent in $G \circ H$ iff: $\left[s_{1}=s_{2}\right.$ and $t_{1}$ is adjacent to $\left.t_{2}\right]$ or $\left[t_{1}=t_{2}\right.$ and $s_{1}$ is adjacent to $s_{2}$ ] or [ $s_{1}$ is adjacent to $s_{2}$ and $t_{1}$ is adjacent to $t_{2}$ ]. Now, the $\Phi$-sum graph under the operation of strong product is defined in [16].

Let $G$ and $H$ be two graphs. For $\Phi \in\{S, R, Q, T\}, \Phi(G)$ is a graph constructed by the operation $\Phi$ on $G$ with set of vertex $V(\Phi(G))$ and set of edge $E(\Phi(G))$. Then, $\Phi$-sum graph $G_{\Phi} \otimes H$ under the strong product of graphs $\Phi(G)$ and $H$ is a graph with vertex set

$$
V\left(G_{\Phi} \otimes H\right)=V(\Phi(G)) \times V(H)=(V(G) \cup E(G)) \times V(H) .
$$

such that two vertex $\left(s_{1}, t_{1}\right)$ and $\left(s_{2}, t_{2}\right)$ of $V\left(G_{\Phi} \otimes H\right)$ are adjacent iff, either $\left[s_{1}=s_{2} \in V(G)\right.$ and $\left.t_{1} t_{2} \in E(H)\right]$ or $\left[t_{1}=t_{2} \in V(H)\right.$ and $\left.s_{1} s_{2} \in E(\Phi(G))\right]$ or $\left[s_{1} s_{2} \in E(\Phi(G))\right.$ and $\left.t_{1} t_{2} \in E(H)\right]$.

It is observed that $G_{\Phi} \otimes H$ has $|V(H)|$ copies of $\Phi(G)$ which are labeled by the vertices of $H$. Also, $V(G)$ and $E(G)$ are shown as blue and red vertices in $G_{\Phi} \otimes H$ respectively, see Figure 2.

\section{Main Results}

Now, we compute the analytical closed expression for FGZ index of the $G_{S} \otimes H, G_{R} \otimes H, G_{Q} \otimes H$ and $G_{T} \otimes H$.

Theorem 1. Let $G$ and $H$ be two graphs. For $\alpha \in N^{+}$and $n=\alpha-1, F G Z$ index of S-sum graph is

$$
\begin{aligned}
M^{\alpha}\left(G_{S} \otimes H\right)= & \sum_{m=0}^{n} \sum_{k=0}^{m}\left(\begin{array}{c}
n \\
m
\end{array}\right)\left(\begin{array}{c}
m \\
k
\end{array}\right)\left[M_{1}^{n-m+k}(G) M_{1}^{m+1}(H)\right. \\
& \left.+M_{1}^{\alpha-m+k}(G) M_{1}^{m}(H)+M_{1}^{\alpha-m+k}(G) M_{1}^{m+1}(H)\right] \\
& +2^{n+1} e_{G} \sum_{m=0}^{n}\left(\begin{array}{c}
n \\
m
\end{array}\right) M_{1}^{m}(H)\left[1+M_{1}(H)\right] .
\end{aligned}
$$

Proof. By the definition for any vertex $(u, v) \in V\left(G_{S} \otimes H\right)$, the degree $d(u, v)$ of $(u, v)$ is

$$
\begin{aligned}
& d(u, v)= \begin{cases}d(u)+d(v)+d(u) d(v) & \text { if } u \in V(G) \\
d(u)+d(u) d(v) & \text { if } u \in V(S(G))-V(G)\end{cases} \\
& d(u, v)= \begin{cases}d(u)+d(v)+d(u) d(v) & \text { if } u \in V(G) \\
2+2 d(v) & \text { if } u \in V(S(G))-V(G),\end{cases} \\
& M^{\alpha}\left(G_{S} \otimes H\right)=\sum_{(u, v) \in V\left(G_{S} \otimes H\right)} d_{G_{S} \otimes H}^{\alpha}(u, v)=\sum_{\left(s_{1}, t_{1}\right)} \sum_{\left(s_{2}, t_{2}\right) \in E\left(G_{S} \otimes H\right)}\left[d^{n}\left(s_{1}, t_{1}\right)+d^{n}\left(s_{2}, t_{2}\right)\right] \\
& =\sum_{s_{1}=s_{2} \in V(G)} \sum_{\left(t_{1} t_{2}\right) \in E(H)}\left[d^{n}\left(s_{1}, t_{1}\right)+d^{n}\left(s_{2}, t_{2}\right)\right]+\sum_{t_{1}=t_{2} \in V(H)} \sum_{\left(s_{1} s_{2}\right) \in E(S(G))}\left[d^{n}\left(s_{1}, t_{1}\right)+d^{n}\left(s_{2}, t_{2}\right)\right] \\
& +\sum_{s_{1} s_{2} \in E(S(G))} \sum_{\left(t_{1} t_{2}\right) \in E(H)}\left[d^{n}\left(s_{1}, t_{1}\right)+d^{n}\left(s_{2}, t_{2}\right)\right]=\sum_{1}+\sum_{2}+\sum_{3} \text {. }
\end{aligned}
$$

For each vertex $s_{1}=s_{2} \in V(G)$ and edge $\left(t_{1} t_{2}\right) \in E(H)$, we have 


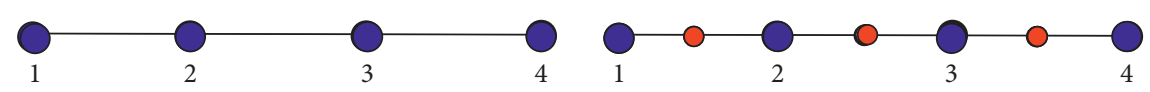

(b)
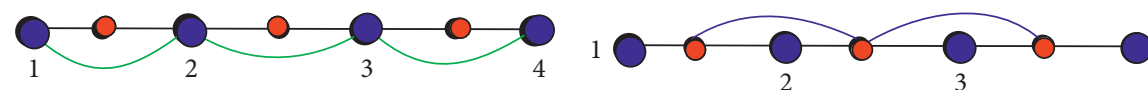

(c)

(d)

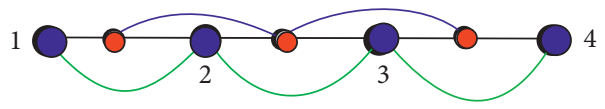

(e)

Figure 1: (a) $G \cong P_{4}$, (b) $S(G)$, (c) $R(G)$, (d) $Q(G)$, and (e) $T(G)$.

G
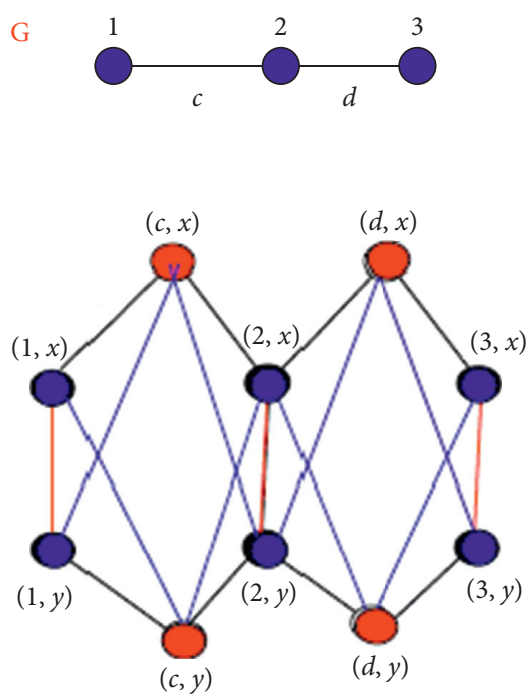

(a)

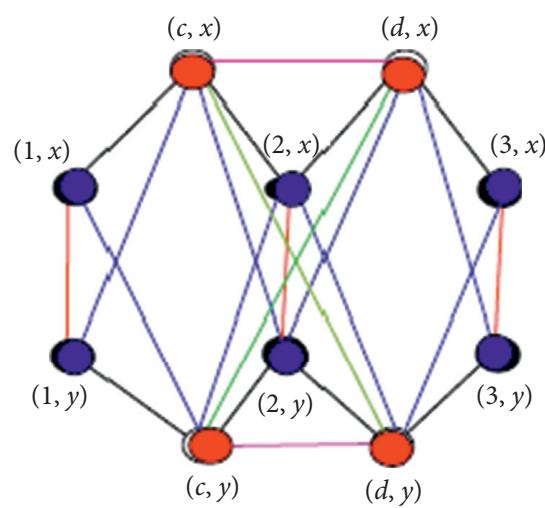

(c)
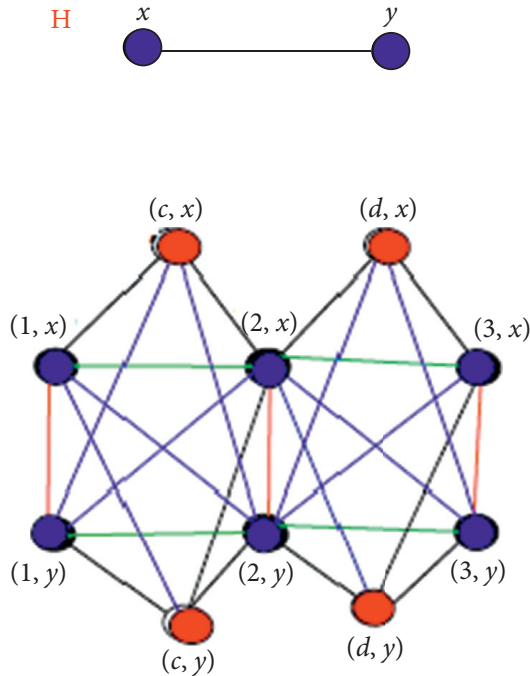

(b)

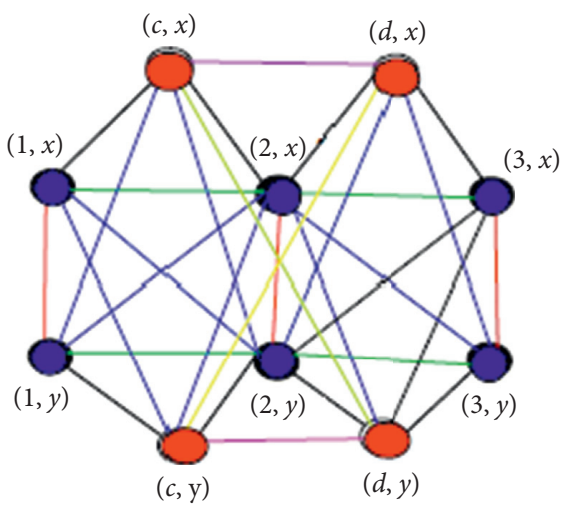

(d)

Figure 2: For $G \cong P_{3}$ and $H \cong P_{2}$, we have (a) $P_{3_{S}} \otimes P_{2}$, (b) $P_{3_{R}} \otimes P_{2}$, (c) $P_{3_{Q}} \otimes P_{2}$, and (d) $P_{3_{T}} \otimes P_{2}$. 


$$
\begin{aligned}
\sum_{1} & =\sum_{s_{1}=s_{2} \in V(G)} \sum_{\left(t_{1} t_{2}\right) \in E(H)}\left[d^{n}\left(s_{1}, t_{1}\right)+d^{n}\left(s_{2}, t_{2}\right)\right] \\
& =\sum_{s \in V(G)} \sum_{\left(t_{1} t_{2}\right) \in E(H)}\left[\left\{d(s)+d\left(t_{1}\right)+d(s) d\left(t_{1}\right)\right\}^{n}+\left\{d(s)+d\left(t_{2}\right)+d(s) d\left(t_{2}\right)\right\}^{n}\right] \\
& =\sum_{s \in V(G)} \sum_{\left(t_{1} t_{2}\right) \in E(H)} \sum_{m=0}^{n} \sum_{k=0}^{m}\left(\begin{array}{c}
n \\
m
\end{array}\right)\left(\begin{array}{c}
m \\
k
\end{array}\right)\left[d^{n-m}(s) d^{m-k}\left(t_{1}\right) d^{k}(s) d^{k}\left(t_{1}\right)+d^{n-m}(s) d^{m-k}\left(t_{2}\right) d^{k}(s) d^{k}\left(t_{2}\right)\right] \\
& =\sum_{s \in V(G)} \sum_{\left(t_{1} t_{2}\right) \in E(H)} \sum_{m=0}^{n} \sum_{k=0}^{m}\left(\begin{array}{c}
n \\
m
\end{array}\right)\left(\begin{array}{c}
m \\
k
\end{array}\right)\left[d^{n-m+k}(s) d^{k}\left(t_{1}\right)+d^{n-m+k}(s) d^{m}\left(t_{2}\right)\right] \\
& =\sum_{s \in V(G)} \sum_{\left(t_{1} t_{2}\right) \in E(H)} \sum_{m=0}^{n} \sum_{k=0}^{m}\left(\begin{array}{c}
n \\
m
\end{array}\right)\left(\begin{array}{c}
m \\
k
\end{array}\right) d^{n-m+k}(s)\left[d^{m}\left(t_{1}\right)+d^{m}\left(t_{2}\right)\right], \\
\sum_{1} & =\sum_{m=0}^{n} \sum_{k=0}^{m}\left(\begin{array}{c}
n \\
m
\end{array}\right)\left(\begin{array}{c}
m \\
k
\end{array}\right) M_{1}^{n-m+k}(G) M_{1}^{m+1}(H) .
\end{aligned}
$$

We know that $|E(S(G))|=2|E(G)|$. Also, for every $t_{1}=$ $t_{2} \in V(H)$ and $\left(s_{1} s_{2}\right) \in E(S(G))$ with $s_{1} \in V(G), s_{2} \in$ $V(S(G))-V(G)$ then,

$$
\begin{aligned}
& \sum_{2}=\sum_{t_{1}=t_{2} \in V(H)} \sum_{\left(s_{1} s_{2}\right) \in E(S(G))}\left[d^{n}\left(s_{1}, t_{1}\right)+d^{n}\left(s_{2}, t_{2}\right)\right] \\
& =\sum_{t \in V(H)} \sum_{\left(s_{1} s_{2}\right) \in E(S(G))}\left[\left\{d\left(s_{1}\right)+d(t)+d\left(s_{1}\right) d(t)\right\}^{n}+\left\{d\left(s_{2}\right)+d\left(s_{2}\right) d(t)\right\}^{n}\right] \\
& =\sum_{t \in V(H)} \sum_{\left(s_{1} s_{2}\right) \in E(S(G))}\left[\sum_{m=0}^{n} \sum_{k=0}^{m}\left(\begin{array}{c}
n \\
m
\end{array}\right)\left(\begin{array}{c}
m \\
k
\end{array}\right)\left\{d^{n-m}\left(s_{1}\right) d^{m-k}(t) d^{k}\left(s_{1}\right) d^{k}(t)\right\}+\sum_{m=0}^{n}\left(\begin{array}{c}
n \\
m
\end{array}\right)\left\{d^{n-m}\left(s_{2}\right) d^{m}\left(s_{2}\right) d^{m}(t)\right\}\right] \\
& =\sum_{t \in V(H)} \sum_{\left(s_{1} s_{2}\right) \in E(S(G))} \sum_{m=0}^{n}\left(\begin{array}{c}
n \\
m
\end{array}\right)\left[\sum_{k=0}^{m}\left(\begin{array}{c}
m \\
k
\end{array}\right) d^{n-m+k}\left(s_{1}\right) d^{m}(t)+2^{n-m}(2 d(t))^{m}\right] \\
& =\sum_{t \in V(H)} \sum_{\left(s_{1} s_{2}\right) \in E(S(G))} \sum_{m=0}^{n}\left(\begin{array}{c}
n \\
m
\end{array}\right)\left[\sum_{k=0}^{m}\left(\begin{array}{c}
m \\
k
\end{array}\right) d^{n-m+k}\left(s_{1}\right) d^{m}(t)+2^{n-m+m} d^{m}(t)\right] \\
& =\sum_{m=0}^{n} \sum_{k=0}^{m}\left(\begin{array}{c}
n \\
m
\end{array}\right)\left(\begin{array}{c}
m \\
k
\end{array}\right)\left\{M_{1}^{n-m+k+1}(G)\right\}\left\{M_{1}^{m}(H)\right\}+\sum_{m=0}^{n}\left(\begin{array}{c}
n \\
m
\end{array}\right)\left\{2^{n+1} e_{G} M_{1}^{m}(H)\right\} \\
& =\sum_{m=0}^{n} \sum_{k=0}^{m}\left(\begin{array}{c}
n \\
m
\end{array}\right)\left(\begin{array}{c}
m \\
k
\end{array}\right) M_{1}^{\alpha-m+k}(G) M_{1}^{m}(H)+e_{G} \sum_{m=0}^{n}\left(\begin{array}{c}
n \\
m
\end{array}\right) 2^{n+1} M_{1}^{m}(H) \text {. } \\
& \sum_{3}=\sum_{s_{1} s_{2} \in E(S(G))} \sum_{\left(t_{1} t_{2}\right) \in E(H)}\left[d^{n}\left(s_{1}, t_{1}\right)+d^{n}\left(s_{2}, t_{2}\right)\right]=\sum_{\substack{s_{1} \in V(G) \\
s_{2} \in V(S(G))-V(G)}} \sum_{\left(t_{1} t_{2}\right) \in E(H)}\left[d^{n}\left(s_{1}, t_{1}\right)+d^{n}\left(s_{2}, t_{2}\right)\right] \\
& =\sum_{\substack{s_{1} \in V(G), s_{2} \in V(S(G))-V(G)}} \sum_{\left(t_{1} t_{2}\right) \in E(H)}\left[\left\{d\left(s_{1}\right)+d\left(t_{1}\right)+d\left(s_{1}\right) d\left(t_{1}\right)\right\}^{n}+\left\{d\left(s_{2}\right)+d\left(s_{2}\right) d\left(t_{2}\right)\right\}^{n}\right] \\
& =\sum_{\substack{s_{1} \in V(G), s_{2} \in V(S(G))-V(G)}} \sum_{\left(t_{1} t_{2}\right) \in E(H)}\left[\sum_{m=0}^{n} \sum_{k=0}^{m}\left(\begin{array}{c}
n \\
m
\end{array}\right)\left(\begin{array}{c}
m \\
k
\end{array}\right)\left\{d^{n-m}\left(s_{1}\right) d^{m-k}\left(t_{1}\right) d^{k}\left(s_{1}\right) d^{k}\left(t_{1}\right)\right\}+\sum_{m=0}^{n}\left(\begin{array}{c}
n \\
m
\end{array}\right)\left\{d^{n-m}\left(s_{2}\right) d^{m}\left(s_{2}\right) d^{m}\left(t_{2}\right)\right\}\right] \\
& =\sum_{\substack{s_{1} \in V(G), s_{2} V(S(G))-V(G)}} \sum_{\left(t_{1} t_{2}\right) \in E(H)} \sum_{m=0}^{n}\left(\begin{array}{c}
n \\
m
\end{array}\right)\left[\sum_{k=0}^{m}\left(\begin{array}{c}
m \\
k
\end{array}\right) d^{n-m+k}\left(s_{1}\right) d^{m-k+k}\left(t_{1}\right)+2^{n-m} 2^{m} d^{m}\left(t_{2}\right)\right] \\
& =\sum_{m=0}^{n}\left(n\left[\sum_{k=0}^{m}\left(\begin{array}{c}
m \\
k
\end{array}\right) \sum_{\substack{s_{1} \in V(G), s_{2} \in V(S(G))-V(G)}} d^{n-m+k}\left(s_{1}\right) \sum_{\left(t_{1} t_{2}\right) \in E(H)} d^{m}\left(t_{1}\right)+2^{n}\left(2 e_{G}\right) \sum_{\left(t_{1} t_{2}\right) \in E(H)} d^{m}\left(t_{2}\right)\right]\right) \\
& =\sum_{m=0}^{n} \sum_{k=0}^{m}\left(\begin{array}{c}
n \\
m
\end{array}\right)\left(\begin{array}{c}
m \\
k
\end{array}\right) M_{1}^{\alpha-m+k}(G) M_{1}^{m+1}(H)+e_{G} \sum_{m=0}^{n}\left(\begin{array}{c}
n \\
m
\end{array}\right) 2^{n+1} M_{1}^{m+1}(H) .
\end{aligned}
$$


Hence

$$
\begin{aligned}
M^{\alpha}\left(G_{S} \otimes H\right)= & \sum_{m=0}^{n} \sum_{k=0}^{m}\left(\begin{array}{c}
n \\
m
\end{array}\right)\left(\begin{array}{c}
m \\
k
\end{array}\right)\left[M_{1}^{n-m+k}(G) M_{1}^{m+1}(H)+M_{1}^{\alpha-m+k}(G) M_{1}^{m}(H)+M_{1}^{\alpha-m+k}(G) M_{1}^{m+1}(H)\right] \\
& +2^{n+1} e_{G} \sum_{m=0}^{n}\left(\begin{array}{c}
n \\
m
\end{array}\right) M_{1}^{m}(H)\left[1+M_{1}(H)\right] .
\end{aligned}
$$

Theorem 2. Let $G$ and $H$ be two graphs. For $\alpha \in N^{+}$and $n=\alpha-1$, FGZ index of $R$-sum graph is

$$
\begin{aligned}
M^{\alpha}\left(G_{R} \otimes H\right)= & \sum_{m=0}^{n} \sum_{k=0}^{m}\left(\begin{array}{c}
n \\
m
\end{array}\right)\left(\begin{array}{c}
m \\
k
\end{array}\right) 2^{n-m+k} M_{1}^{m}(H)\left[M_{1}^{n-m+k}(G) M_{1}(H)+M_{1}^{\alpha-m+k}(G)\left(2+M_{1}(H)\right)\right] \\
& +2 \sum_{m=0}^{n}\left(\begin{array}{c}
n \\
m
\end{array}\right) 2^{n+1} e_{G} M_{1}^{m+1}(H) .
\end{aligned}
$$

Proof. By definition

$$
\begin{aligned}
M^{\alpha}\left(G_{R} \otimes H\right)= & \sum_{(u, v) \in V\left(G_{R} \otimes H\right)} d^{\alpha}(u, v)=\sum_{\left(s_{1}, t_{1}\right)\left(s_{2}, t_{2}\right) \in E\left(G_{R} \otimes H\right)}\left[d^{n}\left(s_{1}, t_{1}\right)+d^{n}\left(s_{2}, t_{2}\right)\right] \\
= & \sum_{s_{1}=s_{2} \in V(G)} \sum_{\left(t_{1} t_{2}\right) \in E(H)}\left[d^{n}\left(s_{1}, t_{1}\right)+d^{n}\left(s_{2}, t_{2}\right)\right]+\sum_{t_{1}=t_{2} \in V(H)}\left(\sum_{\left(s_{1} s_{2}\right) \in E(S(G))}\left[d^{n}\left(s_{1}, t_{1}\right)+d^{n}\left(s_{2}, t_{2}\right)\right]\right) \\
& +\sum_{\left(s_{1} s_{2}\right) \in E(S(G))} \sum_{\left(t_{1} t_{2}\right) \in E(H)}\left[d^{n}\left(s_{1}, t_{1}\right)+d^{n}\left(s_{2}, t_{2}\right)\right]=\sum_{1}+\sum_{2}+\sum_{3} .
\end{aligned}
$$

Consider,

$$
\sum_{1}=\sum_{s_{1}=s_{2} \in V(G)} \sum_{\left(t_{1} t_{2}\right) \in E(H)}\left[d^{n}\left(s_{1}, t_{1}\right)+d^{n}\left(s_{2}, t_{2}\right)\right]=\sum_{m=0}^{n} \sum_{k=0}^{m}\left(\begin{array}{c}
n \\
m
\end{array}\right)\left(\begin{array}{c}
m \\
k
\end{array}\right) 2^{n-m+k} M_{1}^{n-m+k}(G) M_{1}^{m+1}(H)
$$

Since $d_{R(G)}(s)=2 d_{G}(s)$ For each vertex $t_{1}=t_{2} \in E(H)$ and edge $s_{1} s_{2} \in E(R(G))$ where $s_{1}, s_{2} \in(V(G))$ then, 


$$
\begin{aligned}
& \sum_{2}=\sum_{t_{1}=t_{2} \in V(H)} \sum_{s_{1} s_{2} \in E(R(G))}\left[d^{n}\left(s_{1}, t_{1}\right)+d^{n}\left(s_{2}, t_{2}\right)\right]=\sum_{t_{1}=t_{2} \in V(H)} \sum_{\substack{s_{1} \varepsilon_{2} \in E(R(G)), s_{1}, s_{2} \in V(G)}}\left[d^{n}\left(s_{1}, t_{1}\right)+d^{n}\left(s_{2}, t_{2}\right)\right] \\
& +\sum_{t_{1}=t_{2} \in V(H)} \sum_{\substack{s_{1}, s_{2} \in E(R(G)), s_{1} \in V(G), s_{2} \in V(R(G))-V(G)}}\left[d^{n}\left(s_{1}, t_{1}\right)+d^{n}\left(s_{2}, t_{2}\right)\right]=\sum_{2}^{\prime}+\sum_{2}^{\prime \prime} \\
& \sum_{2}^{\prime}=\sum_{t_{1}=t_{2} \in V(H)} \sum_{s_{1} s_{2} \in E(R(G))}\left[d^{n}\left(s_{1}, t_{1}\right)+d^{n}\left(s_{2}, t_{2}\right)\right] \\
& =\sum_{t \in V(H)} \sum_{\left(s_{1} s_{2}\right) \in E(R(G))}\left[\left\{d\left(s_{1}\right)+d(t)+d\left(s_{1}\right) d(t)\right\}^{n}+\left\{d\left(s_{2}\right)+d(t)+d\left(s_{2}\right) d(t)\right\}^{n}\right] \\
& =\sum_{t \in V(H)} \sum_{\left(s_{1} s_{2}\right) \in E(R(G))} \sum_{m=0}^{n} \sum_{k=0}^{m}\left(\begin{array}{c}
n \\
m
\end{array}\right)\left(\begin{array}{c}
m \\
k
\end{array}\right)\left[d^{n-m}\left(s_{1}\right) d^{m-k}(t)\left(d\left(s_{1}\right) d(t)\right)^{k}+d^{n-m}\left(s_{2}\right) d^{m-k}(t)\left(d\left(s_{2}\right) d(t)\right)^{k}\right] \\
& =\sum_{t \in V(H)} \sum_{\substack{\left(s_{1} s_{2}\right) \in E(G) s_{1} \\
s_{2} \in V(G)}} \sum_{m=0}^{n} \sum_{k=0}^{m}\left(\begin{array}{c}
n \\
m
\end{array}\right)\left(\begin{array}{c}
m \\
k
\end{array}\right)(2)^{n-m+k}\left[d^{n-m+k}\left(s_{1}\right) d^{m}(t)+d^{n-m+k}\left(s_{2}\right) d^{m}(t)\right] \\
& =\sum_{m=0}^{n} \sum_{k=0}^{m}\left(\begin{array}{c}
n \\
m
\end{array}\right)\left(\begin{array}{c}
m \\
k
\end{array}\right) 2^{n-m+k} M_{1}^{\alpha-m+k}(G) M_{1}^{m}(H) .
\end{aligned}
$$

For each $t_{1}=t_{2} \in E(H)$ and $s_{1} s_{2} \in E(R(G))$ where $s_{1} \in(V(G)), s_{2} \in(V(R(G))-V(G))$ then,

$$
\begin{aligned}
\sum_{2}^{\prime \prime} & =\sum_{t_{1}=t_{2} \in V(H)} \sum_{\left(s_{1} s_{2}\right) \in E(R(G))}\left[d^{n}\left(s_{1}, t_{1}\right)+d^{n}\left(s_{2}, t_{2}\right)\right] \\
& =\sum_{t \in E(H)} \sum_{\left(s_{1} s_{2}\right) \in E(R(G))}\left[\left\{d\left(s_{1}\right)+d(t)+d\left(s_{1}\right)(t)\right\}^{n}+\left\{d\left(s_{2}\right)+d\left(s_{2}\right) d(t)\right\}^{n}\right] \\
& =\sum_{t \in V(H)} \sum_{\left(s_{1} s_{2}\right) \in E(R(G))} \sum_{m=0}^{n}\left(\begin{array}{c}
n \\
m
\end{array}\right)\left[\sum_{k=0}^{m}\left(\begin{array}{c}
m \\
k
\end{array}\right)\left\{d^{n-m}\left(s_{1}\right) d^{m-k}(t)\left(d\left(s_{1}\right) d(t)\right)^{k}\right\}+\left\{d^{n-m}\left(s_{2}\right)\left(d\left(s_{2}\right) d(t)\right)^{m}\right\}\right] \\
& =\sum_{t \in V(H)} \sum_{s_{1} \in V(G)} \sum_{m=0}^{n}\left(\begin{array}{c}
n \\
m
\end{array}\right)\left[\sum_{k=0}^{m}\left(\begin{array}{c}
m \\
k
\end{array}\right)\left\{2^{n-m+k} d^{n-m+k}\left(s_{1}\right) d^{m}(t)+2^{n-m} 2^{m} d^{m}(t)\right\}\right] \\
& =\sum_{t \in V(H)} \sum_{s_{1} \in(V(G))} \sum_{m=0}^{n}\left(\begin{array}{c}
n \\
m
\end{array}\right)\left[\sum_{k=0}^{m}\left(\begin{array}{c}
m \\
k
\end{array}\right)\left\{2^{n-m+k} d^{n-m+k}\left(s_{1}\right) d^{m}(t)\right\}+\left\{2^{n} d^{m}(t)\right\}\right] \\
& \left.=\sum_{m=0}^{n}\left(\begin{array}{c}
n \\
m
\end{array}\right)\left[\begin{array}{c}
m \\
m
\end{array}\right) 2_{k=0}^{n-m+k} \sum_{t \in V(H)} d^{m}(t) \sum_{s_{1} \in(V(G))} d^{n-m+k}\left(s_{1}\right)+2^{n} \sum_{t \in E(H)} d^{m}(t)\left(2 e_{G}\right)\right] \\
& =\sum_{m=0}^{n} \sum_{k=0}^{m}\left(\begin{array}{c}
n \\
m
\end{array}\right)\left(\begin{array}{c}
m \\
k
\end{array}\right) 2^{n-m+k} M_{1}^{m}(H) M_{1}^{\alpha-m+k}(G)+2^{n+1} e_{G} \sum_{m=0}^{n}\left(\begin{array}{c}
n \\
m
\end{array}\right) M_{1}^{m+1}(H) .
\end{aligned}
$$

For each $t_{1} t_{2} \in E(H)$ and $\left(s_{1} s_{2}\right) \in E(R(G))$, where $s_{1}, s_{2} \in V(G)$ then, 


$$
\begin{aligned}
& \sum_{3}=\sum_{s_{1} s_{2} \in E(R(G))} \sum_{\left(t_{1} t_{2}\right) \in E(H)}\left[d^{n}\left(s_{1}, t_{1}\right)+d^{n}\left(s_{2}, t_{2}\right)\right] \\
& \sum_{3}=\sum_{\substack{s_{1} s_{2} \in E(R(G)) \\
s_{1}, s_{2} \in V(G)}} \sum_{\left(t_{1} t_{2}\right) \in E(H)}\left[d^{n}\left(s_{1}, t_{1}\right)+d^{n}\left(s_{2}, t_{2}\right)\right]+\sum_{\substack{s_{1} s_{1} \in E(R(G)), s_{1} \in V(G) \\
s_{2} \in V(R(G))-V(G)}} \sum_{\substack{\left.t_{1} t_{2}\right) \in E(H) \\
s_{2} \in\left(s^{\prime}\right)}}\left[d^{n}\left(s_{1}, t_{1}\right)+d^{n}\left(s_{2}, t_{2}\right)\right] \\
& =\sum_{\left(t_{1} t_{2}\right) \in E(H)} \sum_{s_{1}, s_{2} \in V} \sum_{(G)}^{n} \sum_{m=0}^{m}\left(\begin{array}{c}
n \\
m
\end{array}\right)\left(\begin{array}{c}
m \\
k
\end{array}\right)\left[\left\{\left(2 d\left(s_{1}\right)\right)^{n-m+k} d^{m}\left(t_{1}\right)\right\}+\left\{\left(2 d\left(s_{2}\right)\right)^{n-m+k} d^{m}\left(t_{2}\right)\right\}\right] \\
& =\sum_{\left(t_{1} t_{2}\right) \in E(H)} \sum_{s_{1}, s_{2} \in V(G)} \sum_{m=0}^{n} \sum_{k=0}^{m}\left(\begin{array}{c}
n \\
m
\end{array}\right)\left(\begin{array}{c}
m \\
k
\end{array}\right) 2^{n-m+k}\left[d^{n-m+k}\left(s_{1}\right) d^{m}\left(t_{1}\right)+d^{n-m+k}\left(s_{2}\right) d^{m}\left(t_{2}\right)\right]=\sum_{3}^{\prime}+\sum_{3}^{\prime \prime}, \\
& \sum_{3}^{\prime}=\sum_{\left(t_{1} t_{2}\right) \in E(H)} \sum_{\left(s_{1} s_{2}\right) \in E(R(G))}\left[d^{n}\left(s_{1}, t_{1}\right)+d^{n}\left(s_{2}, t_{2}\right)\right] \\
& =\sum_{\left(t_{1} t_{2}\right) \in E(H)} \sum_{\left(s_{1} s_{2}\right) \in E(R(G))}\left[\left\{d\left(s_{1}\right)+d\left(t_{1}\right)+d\left(s_{1}\right) d\left(t_{1}\right)\right\}^{n}+\left\{d\left(s_{2}\right)+d\left(t_{2}\right)+d\left(s_{2}\right) d\left(t_{2}\right)\right\}^{n}\right] \\
& =\sum_{\left(t_{1} t_{2}\right) \in E(H)} \sum_{\left(s_{1} s_{2}\right) \in E(R(G))} \sum_{m=0}^{n} \sum_{k=0}^{m}\left(\begin{array}{c}
n \\
m
\end{array}\right)\left(\begin{array}{c}
m \\
k
\end{array}\right)\left[\left\{d^{n-m+k}\left(s_{1}\right) d^{m}\left(t_{1}\right)\right\}+\left\{d^{n-m+k}\left(s_{2}\right) d^{m}\left(t_{2}\right)\right\}\right] \\
& =\sum_{m=0}^{n} \sum_{k=0}^{m}\left(\begin{array}{c}
n \\
m
\end{array}\right)\left(\begin{array}{c}
m \\
k
\end{array}\right) 2^{n-m+k} M_{1}^{\alpha-m+k}(G) M_{1}^{m+1}(H) \text {. }
\end{aligned}
$$

For each $t_{1} t_{2} \in E(H)$ and $\left(s_{1} s_{2}\right) \in E(R(G))$, where $s_{1} \in V(G), s_{2} \in V(R(G))-V(G)$ then,

$$
\begin{aligned}
\sum_{3}^{\prime \prime} & =\sum_{t_{1} t_{2} \in E(H)} \sum_{\left(s_{1} s_{2}\right) \in E(R(G))}\left[d^{n}\left(s_{1}, t_{1}\right)+d^{n}\left(s_{2}, t_{2}\right)\right] \\
& =\sum_{t_{1} t_{2} \in E(H)} \sum_{\left(s_{1} s_{2}\right) \in E(R(G))}\left[\left\{d\left(s_{1}\right)+d\left(t_{1}\right)+d\left(s_{1}\right) d\left(t_{1}\right)\right\}^{n}+\left\{2+2 d\left(t_{2}\right)\right\}^{n}\right] \\
& =\sum_{t_{1} t_{2} \in E(H)} \sum_{\left(s_{1} s_{2}\right) \in E(R(G))} \sum_{m=0}^{n}\left(\begin{array}{c}
n \\
m
\end{array}\right)\left[\sum_{k=0}^{m}\left(\begin{array}{l}
m \\
k
\end{array}\right) d^{n-m}\left(s_{1}\right) d^{m-k}\left(t_{1}\right)\left(d\left(s_{1}\right) d\left(t_{1}\right)\right)^{k}+2^{n-m}\left(2 d\left(t_{2}\right)\right)^{m}\right] \\
& =\sum_{t_{1} t_{2} \in E(H)} \sum_{s_{1}, s_{2} \in V(G)} \sum_{m=0}^{n}\left(\begin{array}{c}
n \\
m
\end{array}\right)\left[\sum_{k=0}^{m}\left(\begin{array}{c}
m \\
k
\end{array}\right)\left(2 d\left(s_{1}\right)\right)^{n-m+k} d^{m}\left(t_{1}\right)+2^{n-m} 2 d\left(t_{2}\right)^{m}\right] \\
& =\sum_{t_{1} t_{2} \in E(H)} \sum_{s_{1}, s_{2} \in V(G)} \sum_{m=0}^{n}\left(\begin{array}{c}
n \\
m
\end{array}\right)\left[\sum_{k=0}^{m}\left(\begin{array}{c}
m \\
k
\end{array}\right) 2^{n-m+k} d^{n-m+k}\left(s_{1}\right) d^{m}\left(t_{1}\right)+2^{n} d^{m}\left(t_{2}\right)\right] \\
& =\sum_{m=0}^{n}\left(\begin{array}{c}
n \\
m
\end{array}\right)\left[\sum_{k=0}^{m}\left(\begin{array}{c}
m \\
k
\end{array}\right) 2^{n-m+k} \sum_{s_{1}, s_{2} \in V(G)} d_{G}^{n-m+k}\left(s_{1}\right) \sum_{t_{1} t_{2} \in E(H)} d^{m}\left(t_{1}\right)+2^{n} \sum_{t_{1} t_{2} \in E(H)} d^{m}\left(t_{2}\right)\left(2 e_{G}\right)\right] \\
& =\sum_{m=0}^{n}\left(\begin{array}{c}
n \\
m
\end{array}\right)\left[\sum_{k=0}^{m}\left(\begin{array}{c}
m \\
k
\end{array}\right) 2^{n-m+k} M_{1}^{\alpha-m+k}(G) M_{1}^{m+1}(H)+2^{n+1} e_{G} M_{1}^{m+1}(H)\right] \\
\sum_{3}^{\prime \prime} & =\sum_{m=0}^{n}\left(\begin{array}{c}
n \\
m
\end{array}\right)\left[\sum_{k=0}^{m}\left(\begin{array}{c}
m \\
k
\end{array}\right) 2^{n-m+k} M_{1}^{\alpha-m+k}(G) M_{1}^{m+1}(H)+2^{n+1} e_{G} M_{1}^{m+1}(H) .\right.
\end{aligned}
$$


Hence

$$
\begin{aligned}
M^{\alpha}\left(G_{R} \otimes H\right)= & \sum_{m=0}^{n} \sum_{k=0}^{m}\left(\begin{array}{c}
n \\
m
\end{array}\right)\left(\begin{array}{c}
m \\
k
\end{array}\right) 2^{n-m+k} M_{1}^{m}(H)\left[M_{1}^{n-m+k}(G) M_{1}(H)+M_{1}^{\alpha-m+k}(G)\left(2+M_{1}(H)\right)\right] \\
& +2 \sum_{m=0}^{n}\left(\begin{array}{c}
n \\
m
\end{array}\right) 2^{n+1} e_{G} M_{1}^{m+1}(H) .
\end{aligned}
$$

Theorem 3. Let $G$ and $H$ be two graphs. For $\alpha \in N^{+}$and $n=\alpha-1, F G Z$ index of Q-sum graph is

$$
\begin{aligned}
M^{\alpha}\left(G_{Q} \otimes H\right)= & \sum_{m=0}^{n} \sum_{k=0}^{m}\left(\begin{array}{c}
n \\
m
\end{array}\right)\left(\begin{array}{c}
m \\
k
\end{array}\right) M_{1}^{n+k-m}(G) M_{1}^{m}(H)\left[M_{1}(H)+M_{1}(G)+M_{1}(G) M_{1}(H)\right] \\
& +\sum_{m=0}^{n} \sum_{i=0}^{n}\left(\begin{array}{c}
n \\
m
\end{array}\right)\left(\begin{array}{c}
n \\
i
\end{array}\right) \sum_{u v \in E(G)} M_{1}^{m}(H)\left[d_{G}^{n-i}(u) d_{G}^{i}(v)\right]\left[2+2 M_{1}(H)\right]+\sum_{m=0}^{n}\left(\begin{array}{c}
n \\
m
\end{array}\right) \sum_{i=0}^{n}\left(\begin{array}{c}
n \\
i
\end{array}\right) \\
& \cdot \sum_{\substack{u v, v w \\
\in E(G)}} M_{1}^{m}(H)\left[d_{G}^{n-i}(u) d_{G}^{i}(v)+d_{G}^{n-i}(v) d_{G}^{i}(w)\right]\left[1+M_{1}(H)\right] .
\end{aligned}
$$

Proof. By definition

$$
\begin{aligned}
M^{\alpha}\left(G_{Q} \otimes H\right)= & \sum_{(u, v) \in V\left(G_{Q} \otimes H\right)} d^{\alpha}(u, v)=\sum_{\left(s_{1}, t_{1}\right)} \sum_{\left(s_{2}, t_{2}\right) \in E\left(G_{Q} \otimes H\right)}\left[d^{n}\left(s_{1}, t_{1}\right)+d^{n}\left(s_{2}, t_{2}\right)\right] \\
= & \sum_{s_{1}=s_{2} \in V(G)} \sum_{t_{1} t_{2} \in E(H)}\left[d^{n}\left(s_{1}, t_{1}\right)+d^{n}\left(s_{2}, t_{2}\right)\right]+\sum_{t_{1} t_{2} \in E(H)} \sum_{s_{1} s_{2} \in E(Q(G))}\left[d^{n}\left(s_{1}, t_{1}\right)+d^{n}\left(s_{2}, t_{2}\right)\right] \\
& +\sum_{s_{1} s_{2} \in E(Q(G))} \sum_{\left(t_{1} t_{2}\right) \in E(H)}\left[d^{n}\left(s_{1}, t_{1}\right)+d^{n}\left(s_{2}, t_{2}\right)\right]=\sum_{1}+\sum_{2}+\sum_{3} .
\end{aligned}
$$

Consider,

$$
\sum_{1}=\sum_{s_{1}=s_{2} \in V(G)} \sum_{\left(t_{1} t_{2}\right) \in E(H)}\left[d^{n}\left(s_{1}, t_{1}\right)+d^{n}\left(s_{2}, t_{2}\right)\right]=\sum_{m=0}^{n} \sum_{k=0}^{m}\left(\begin{array}{c}
n \\
m
\end{array}\right)\left(\begin{array}{c}
m \\
k
\end{array}\right) M_{1}^{n-m+k}(G) M_{1}^{m+1}(H) .
$$

For each vertex $t_{1}=t_{2} \in V(H)$ and edge $s_{1} s_{2} \in E(Q(G))$ where $s_{1} \in V(G), s_{2} \in V(Q(G))-V(G)$. Note that $d_{Q(G)} s_{2}$
$=d_{G}(u)+d_{G}(v)$ for $s_{2} \in[V(Q(G))-V(G)], s_{2}$ is the vertex inserted into the edge $u v$ of $G$ for all $u, v \in V(G)$, we have 


$$
\begin{aligned}
& \sum_{2}=\sum_{t_{1}=t_{2} \in V(H)} \sum_{s_{1} s_{2} \in E(Q(G))}\left[d^{n}\left(s_{1}, t_{1}\right)+d^{n}\left(s_{2}, t_{2}\right)\right] \\
& \sum_{2}=\sum_{t_{1}=t_{2} \in V(H)} \sum_{\substack{\left(s_{1} s_{2}\right) \in E(Q(G)), s_{1} \in V(G) s_{2} \in V(Q(G))-V(G)}}\left[d^{n}\left(s_{1}, t_{1}\right)+d^{n}\left(s_{2}, t_{2}\right)\right] \\
& +\sum_{t_{1}=t_{2} \in V(H)} \sum_{\substack{s_{1} s_{2} \in E(Q(G)), s_{1}, s_{2} \in V(Q(G))-V(G)}}\left[d^{n}\left(s_{1}, t_{1}\right)+d^{n}\left(s_{2}, t_{2}\right)\right]=\sum_{2^{\prime}}+\sum_{2^{\prime \prime}} \\
& \sum_{2^{\prime}}=\sum_{t_{1}=t_{2} \in V(H)} \sum_{\left(s_{1} s_{2}\right) \in E(Q(G))}\left[d^{n}\left(s_{1}, t_{1}\right)+d^{n}\left(s_{2}, t_{2}\right)\right] \\
& =\sum_{t \in V(H)} \sum_{\left(s_{1} s_{2}\right) \in E(Q(G))}\left[\left\{d\left(s_{1}\right)+d(t)+d\left(s_{1}\right) d(t)\right\}^{n}+\left\{d\left(s_{2}\right)+d\left(s_{2}\right) d(t)\right\}^{n}\right] \\
& =\sum_{t \in V(H)} \sum_{\left(s_{1} s_{2}\right) \in E(Q(G))} \sum_{m=0}^{n}\left(\begin{array}{c}
n \\
m
\end{array}\right)\left[\sum_{k=0}^{m}\left(\begin{array}{l}
m \\
k
\end{array}\right) d^{n-m}\left(s_{1}\right) d^{m-k}(t)\left(d\left(s_{1}\right) d(t)\right)^{k}+d^{n-m}\left(s_{2}\right)\left(d\left(s_{2}\right) d(t)\right)^{m}\right] \\
& =\sum_{t \in V(H)} \sum_{\left(s_{1} s_{2}\right) \in E(Q(G))} \sum_{m=0}^{n}\left(\begin{array}{c}
n \\
m
\end{array}\right)\left[\sum_{k=0}^{m}\left(\begin{array}{c}
m \\
k
\end{array}\right) d^{n-m}\left(s_{1}\right) d^{m-k}(t)\left(d\left(s_{1}\right) d(t)\right)^{k}+d^{n}\left(s_{2}\right) d^{m}(t)\right],
\end{aligned}
$$

consider

$$
C_{1}=\sum_{\substack{s_{1} s_{2} \in E(Q(G)), s_{1} \in V(G) s_{2} \in V(Q(G))-V(G)}} d^{n}\left(s_{1}\right) .
$$

Let

In $C_{1}, s_{1} \in V(G)$ and $d^{n}\left(s_{1}\right)$ occurs $d\left(s_{1}\right)$ times. Thus

$$
C_{1}=\sum_{\substack{s_{1} s_{2} \in E(Q(G)), s_{1} \in V(G), s_{2} \in V(Q(G))-V(G)}} d^{n+1}\left(s_{1}\right) .
$$

$$
C_{2}=\sum_{\substack{s_{1} s_{2} \in E(Q(G)), s_{1} \in V(G), s_{2} \in V(Q(G))-V(G)}} d^{n}\left(s_{2}\right) .
$$

In $C_{1}, s_{2}=u v \in E(G)$ and $d^{n}\left(s_{2}\right)$ occurs two times. Therefore

$$
\begin{aligned}
C_{2} & =2 \sum_{\substack{s_{2}=u v \in \\
V(Q(G))-V(G)}}[d(u)+d(v)]^{n}=2 \sum_{u v \in E(G)} \sum_{i=0}^{n}\left(\begin{array}{c}
n \\
i
\end{array}\right) d_{G}^{n-i}(u) d_{G}^{i}(v) \\
\sum_{2^{\prime}} & =\sum_{t \in V(H)} \sum_{\substack{\left.s_{1} s_{2}\right) \in E(Q(G)) \\
m=0}} \sum_{m}^{n}\left(\begin{array}{c}
n \\
m
\end{array}\right)\left[\sum_{k=0}^{m}\left(\begin{array}{c}
m \\
k
\end{array}\right) d^{n-m+k}\left(s_{1}\right) d^{m}(t)+\sum_{t \in V(H)} d^{m}(t) \sum_{\left(s_{1} s_{2}\right) \in E(Q(G))} d^{n}\left(s_{2}\right)\right] \\
& =\sum_{m=0}^{n} \sum_{k=0}^{m}\left(\begin{array}{c}
n \\
m
\end{array}\right)\left(\begin{array}{c}
m \\
k
\end{array}\right) M_{1}^{\alpha-m+k}(G) M_{1}^{m}(H)+2 \sum_{m=0}^{n}\left(\begin{array}{c}
n \\
m
\end{array}\right) M_{1}^{m}(H) \sum_{u v \in G} \sum_{i=0}^{n}\left(\begin{array}{c}
n \\
i
\end{array}\right) d^{n-i}(u) d^{i}(v) .
\end{aligned}
$$

Now $\forall t_{1}=t_{2} \in V(H)$ and edge $s_{1} s_{2} \in E(Q(G))$ where $s_{1}, s_{2} \in V(Q(G))-V(G)$, we have 


$$
\begin{aligned}
& \sum_{2}^{\prime \prime}=\sum_{t_{1}=t_{2} \in V(H)} \sum_{s_{1}, s_{2} \in E(Q(G))}\left[d^{n}\left(s_{1}, t_{1}\right)+d^{n}\left(s_{2}, t_{2}\right)\right] \\
& =\sum_{t \in V(H)} \sum_{s_{1}, s_{2} \in E(Q(G))}\left[\left\{d\left(s_{1}\right)+d\left(s_{1}\right) d(t)\right\}^{n}+\left\{d\left(s_{2}\right)+d\left(s_{2}\right) d(t)\right\}^{n}\right] \\
& =\sum_{t \in V(H)} \sum_{s_{1}, s_{2} \in E(Q(G))}\left[\left\{\sum_{m=0}^{n}\left(\begin{array}{c}
n \\
m
\end{array}\right) d^{n-m}\left(s_{1}\right) d^{m}\left(s_{1}\right) d^{m}(t)\right\}+\left\{\sum_{m=0}^{n}\left(\begin{array}{c}
n \\
m
\end{array}\right) d^{n-m}\left(s_{2}\right) d^{m}\left(s_{2}\right) d^{m}(t)\right\}\right] \\
& =\sum_{t \in V(H)} \sum_{s_{1}, s_{2} \in E(Q(G))}\left[\left\{\sum_{m=0}^{n}\left(\begin{array}{c}
n \\
m
\end{array}\right) d^{n}\left(s_{1}\right) d^{m}(t)\right\}+\left\{\sum_{m=0}^{n}\left(\begin{array}{c}
n \\
m
\end{array}\right) d^{n}\left(s_{2}\right) d^{m}(t)\right\}\right] \\
& =\sum_{t \in V(H)} \sum_{s_{1}, s_{2} \in E(Q(G))} \sum_{m=0}^{n}\left(\begin{array}{c}
n \\
m
\end{array}\right) d^{m}(t)\left[d^{n}\left(s_{1}\right)+d^{n}\left(s_{2}\right)\right] \\
& =\sum_{t \in V(H)} \sum_{u v, v w \in E(G)} \sum_{m=0}^{n}\left(\begin{array}{c}
n \\
m
\end{array}\right) d^{m}(t)\left[(d(u)+d(v))^{n}+(d(v)+d(w))^{n}\right] \\
& =\sum_{t \in V(H)} \sum_{u v, v w \in E(G)} \sum_{m=0}^{n}\left(\begin{array}{c}
n \\
m
\end{array}\right) d^{m}(t)\left[\sum_{i=0}^{n}\left(\begin{array}{c}
n \\
i
\end{array}\right)\left(d^{n-i}(u) d^{i}(v)\right)+\sum_{i=0}^{n}\left(\begin{array}{l}
n \\
i
\end{array}\right) d^{n-i}(v) d^{i}(w)\right] \\
& =\sum_{m=0}^{n}\left(\begin{array}{c}
n \\
m
\end{array}\right) \sum_{t \in V(H)} d^{m}(t) \sum_{u v, v w \in E(G)} \sum_{i=0}^{n}\left(\begin{array}{c}
n \\
i
\end{array}\right)\left[d^{n-i}(u) d^{i}(v)+d^{n-i}(v) d^{i}(w)\right] \\
& =\sum_{m=0}^{n}\left(\begin{array}{c}
n \\
m
\end{array}\right) \sum_{t \in V(H)} d^{m}(t) \sum_{u v, v w \in E(G)} \sum_{i=0}^{n}\left(\begin{array}{l}
n \\
i
\end{array}\right)\left[d^{n-i}(u) d^{i}(v)+d^{n-i}(v) d^{i}(w)\right] \\
& =\sum_{m=0}^{n}\left(\begin{array}{c}
n \\
m
\end{array}\right) \sum_{t \in V(H)} d^{m}(t) \sum_{u v, v w \in E(G)} \sum_{i=0}^{n}\left(\begin{array}{c}
n \\
i
\end{array}\right)\left[d^{n-i}(u) d^{i}(v)+d^{n-i}(v) d^{i}(w)\right] \\
& =\sum_{m=0}^{n}\left(\begin{array}{c}
n \\
m
\end{array}\right) M_{1}^{m}(H) \sum_{u v, v w \in E(G)} \sum_{i=0}^{n}\left(\begin{array}{l}
n \\
i
\end{array}\right)\left[d^{n-i}(u) d^{i}(v)+d^{n-i}(v) d^{i}(w)\right] .
\end{aligned}
$$

For each $t_{1} t_{2} \in E(H)$ and $\left(s_{1} s_{2}\right) \in E(Q(G))$, where $s_{1}, s_{2} \in V(G)$, we have 


$$
\begin{aligned}
& \sum_{3}=\sum_{s_{1} s_{2} \in E(Q(G))} \sum_{\left(t_{1} t_{2}\right) \in E(H)}\left[d^{n}\left(s_{1}, t_{1}\right)+d^{n}\left(s_{2}, t_{2}\right)\right] \\
& \sum_{3}=\sum_{\substack{s_{1} s_{\epsilon} \in E(Q(G)) \\
s_{1} \in V(G), s_{2} \in V(Q(G))-V(G)}} \sum_{\left(t_{1} t_{2}\right) \in E(H)}\left[d^{n}\left(s_{1}, t_{1}\right)+d^{n}\left(s_{2}, t_{2}\right)\right]
\end{aligned}
$$

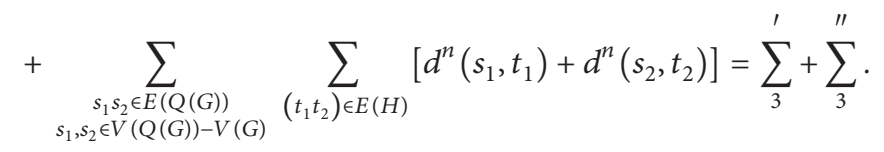

$$
\begin{aligned}
& \sum_{3}^{\prime}=\sum_{t_{1} t_{2} \in E(H)} \sum_{\left(s_{1} s_{2}\right) \in E(Q(G))}\left[d^{n}\left(s_{1}, t_{1}\right)+d^{n}\left(s_{2}, t_{2}\right)\right] \\
& =\sum_{t_{1} t_{2} \in E(H)} \sum_{\left(s_{1} s_{2}\right) \in E(Q(G))}\left[\left\{d\left(s_{1}\right)+d\left(t_{1}\right)+d\left(s_{1}\right) d\left(t_{1}\right)\right\}^{n}+\left\{d\left(s_{2}\right)+d\left(s_{2}\right) d\left(t_{2}\right)\right\}^{n}\right] \\
& =\sum_{t_{1} t_{2} \in E(H)} \sum_{\left(s_{1} s_{2} t \in n E q(Q(G))\right.} \sum_{m=0}^{n}\left(\begin{array}{c}
n \\
m
\end{array}\right)\left[\sum_{k=0}^{m}\left(\begin{array}{c}
m \\
k
\end{array}\right) d^{n-m}\left(s_{1}\right) d^{m-k}\left(t_{1}\right)\left(d\left(s_{1}\right) d\left(t_{1}\right)\right)^{k}+d^{n-m}\left(s_{2}\right)\left(d\left(s_{2}\right) d\left(t_{2}\right)\right)^{m}\right] \\
& =\sum_{t_{1} t_{2} \in E(H)} \sum_{\left(s_{1} s_{2}\right) \in E(Q(G))} \sum_{m=0}^{n}\left(\begin{array}{c}
n \\
m
\end{array}\right)\left[\sum_{k=0}^{m}\left(\begin{array}{c}
m \\
k
\end{array}\right) d^{n-m+k}\left(s_{1}\right) d^{m}\left(t_{1}\right)+d^{n}\left(s_{2}\right) d^{m}\left(t_{2}\right)\right] \\
& =\sum_{m=0}^{n}\left(\begin{array}{c}
n \\
m
\end{array}\right)\left[\sum_{k=0}^{m}\left(\begin{array}{c}
m \\
k
\end{array}\right) M_{1}^{\alpha-m+k}(G) M_{1}^{m+1}(H)+\sum_{t_{1} t_{2} \in E(H)} d^{m}\left(t_{2}\right) \sum_{\left(s_{1} s_{2}\right) \in E(Q(G))} d^{n}\left(s_{2}\right)\right] \\
& =\sum_{m=0}^{n}\left(\begin{array}{c}
n \\
m
\end{array}\right)\left[\sum_{k=0}^{m}\left(\begin{array}{c}
m \\
k
\end{array}\right) M_{1}^{\alpha-m+k}(G) M_{1}^{m+1}(H)+2 \sum_{t_{1} t_{2} \in E(H)} d^{m}\left(t_{2}\right) \sum_{u, v \in V(G)}(d(u)+d(v))^{n}\right] \\
& =\sum_{m=0}^{n}\left(\begin{array}{c}
n \\
m
\end{array}\right)\left[\sum_{k=0}^{m}\left(\begin{array}{c}
m \\
k
\end{array}\right) M_{1}^{\alpha-m+k}(G) M_{1}^{m+1}(H)+2 M_{1}^{m+1}(H) \sum_{u, v \in V(G)}(d(u)+d(v))^{n}\right] \\
& =\sum_{m=0}^{n}\left(\begin{array}{c}
n \\
m
\end{array}\right)\left[\sum_{k=0}^{m}\left(\begin{array}{c}
m \\
k
\end{array}\right) M_{1}^{\alpha-m+k}(G) M_{1}^{m+1}(H)+2 M_{1}^{m+1}(H) \sum_{u, v \in V(G)} \sum_{i=0}^{n} d^{n-i}(u) d^{i}(v)\right] \\
& =\sum_{m=0}^{n}\left(\begin{array}{c}
n \\
m
\end{array}\right)\left[\sum_{k=0}^{m}\left(\begin{array}{c}
m \\
k
\end{array}\right) M_{1}^{\alpha-m+k}(G) M_{1}^{m+1}(H)+2 M_{1}^{m+1}(H) \sum_{i=0}^{n} \sum_{u, v \in V(G)} d^{n-i}(u) d^{i}(v)\right] .
\end{aligned}
$$

For each $t_{1} t_{2} \in E(H)$ and $\left(s_{1} s_{2}\right) \in E(Q(G))$, where $s_{1} \in V(G), s_{2} \in V(Q(G))-V(G)$, we have

$$
\begin{aligned}
\sum_{3}^{\prime \prime} & =\sum_{t_{1} t_{2} \in E(H)} \sum_{\left(s_{1} s_{2}\right) \in E(Q(G))}\left[d^{n}\left(s_{1}, t_{1}\right)+d^{n}\left(s_{2}, t_{2}\right)\right] \\
& =\sum_{t_{1} t_{2} \in E(H)} \sum_{\left(s_{1} s_{2}\right) \in E(Q(G))}\left[\left\{d\left(s_{1}\right)+d\left(s_{1}\right) d\left(t_{1}\right)\right\}^{n}+\left\{d\left(s_{2}\right)+d\left(s_{2}\right) d\left(t_{2}\right)\right\}^{n}\right] \\
& =\sum_{t_{1} t_{2} \in E(H)} \sum_{\left(s_{1} s_{2}\right) \in E(Q(G))}\left[\sum_{m=0}^{n}\left(\begin{array}{c}
n \\
m
\end{array}\right)\left\{d^{n-m}\left(s_{1}\right)\left(d\left(s_{1}\right) d\left(t_{1}\right)\right)^{m}\right\}+\sum_{m=0}^{n}\left(\begin{array}{c}
n \\
m
\end{array}\right)\left\{d^{n-m}\left(s_{2}\right)\left(d\left(s_{2}\right) d\left(t_{2}\right)\right)^{m}\right\}\right] \\
& =\sum_{m=0}^{n}\left(\begin{array}{c}
n \\
m
\end{array}\right) M_{1}^{m+1}(H)\left[\sum_{u, v \in V(G)}(d(u)+d(v))^{n}+\sum_{v, w \in V(G)}(d(v)+d(w))^{n}\right] \\
& =\sum_{m=0}^{n}\left(\begin{array}{c}
n \\
m
\end{array}\right) M_{1}^{m+1}(H) \sum_{i=0}^{n}\left(\begin{array}{c}
n \\
i
\end{array}\right)\left[\sum_{u, v \in V(G)} d^{n-i}(u) d^{i}(v)+\sum_{v, w \in V(G)} d^{n-i}(v)+d^{i}(w)\right] .
\end{aligned}
$$


Hence

$$
\begin{aligned}
M^{\alpha}\left(G_{Q} \otimes H\right)= & \sum_{m=0}^{n} \sum_{k=0}^{m}\left(\begin{array}{c}
n \\
m
\end{array}\right)\left(\begin{array}{l}
m \\
k
\end{array}\right) M_{1}^{n+k-m}(G) M_{1}^{m}(H)\left[M_{1}(H)+M_{1}(G)+M_{1}(G) M_{1}(H)\right] \\
& +\sum_{m=0}^{n} \sum_{i=0}^{n}\left(\begin{array}{c}
n \\
m
\end{array}\right)\left(\begin{array}{c}
n \\
i
\end{array}\right) \sum_{u v \in E(G)} M_{1}^{m}(H)\left[d_{G}^{n-i}(u) d_{G}^{i}(v) 2+2 M_{1}(H)\right]+\sum_{m=0}^{n}\left(\begin{array}{c}
n \\
m
\end{array}\right) \sum_{i=0}^{n}\left(\begin{array}{c}
n \\
i
\end{array}\right) \\
& \cdot \sum_{\substack{u v, v w \\
\in E(G)}} M_{1}^{m}(H)\left[d_{G}^{n-i}(u) d_{G}^{i}(v)+d_{G}^{n-i}(v) d_{G}^{i}(w)\right]\left[1+M_{1}(H)\right] .
\end{aligned}
$$

Theorem 4. Let $G$ and $H$ be two graphs. For $\alpha \in N^{+}$and $n=\alpha-1, F G Z$ index of T-sum graph is

$$
\begin{aligned}
M^{\alpha}\left(G_{T} \otimes H\right)= & \sum_{m=0}^{n} \sum_{k=0}^{m}\left(\begin{array}{c}
n \\
m
\end{array}\right)\left(\begin{array}{c}
m \\
k
\end{array}\right) 2^{n-m+k} M_{1}^{m+1}(H) M_{1}^{k-m}(G)\left[M_{1}^{n}(G)+3 M_{1}^{\alpha}(G)\right] \\
& +\sum_{m=0}^{n}\left(\begin{array}{c}
n \\
m
\end{array}\right) \sum_{u v \in V(G)} \sum_{i=0}^{n}\left(\begin{array}{l}
n \\
i
\end{array}\right)\left[d_{G}^{n-i}(u) d_{G}^{i}(v)\right]\left[3 M_{1}^{m}(H)+3 M_{1}^{m+1}(H)\right] \\
& +\sum_{m=0}^{n}\left(\begin{array}{c}
n \\
m
\end{array}\right) \sum_{u v \in V(G)} \sum_{i=0}^{n}\left(\begin{array}{l}
n \\
i
\end{array}\right)\left[d_{G}^{n-i}(v) d_{G}^{i}(w)\right]\left[2 M_{1}^{m+1}(H)+M_{1}^{m}(H)\right] .
\end{aligned}
$$

Theorem 5. Let $G$ and $H$ be two graphs. Then, the FGZI of $\Phi$-sum graphs where $\alpha \in \mathfrak{R}$, we have

$$
\text { (i) } \begin{aligned}
& M^{\alpha}\left(G_{S} \otimes H\right)=\sum_{m=0}^{\infty} \sum_{k=0}^{\infty}\left(\begin{array}{c}
n \\
m
\end{array}\right)\left(\begin{array}{c}
m \\
k
\end{array}\right)\left[M_{1}^{n-m+k}(G) M_{1}^{m+1}(H)\right. \\
+ & \left.M_{1}^{\alpha-m+k}(G) M_{1}^{m}(H)+M_{1}^{\alpha-m+k}(G) M_{1}^{m+1}(H)\right] \\
+ & 2^{n+1} e_{G} \sum_{m=0}^{\infty}\left(\begin{array}{c}
n \\
m
\end{array}\right) M_{1}^{m}(H)\left[1+M_{1}(H)\right],
\end{aligned}
$$

(ii) $M^{\alpha}\left(G_{R} \otimes H\right)=\sum_{m=0}^{\infty} \sum_{k=0}^{\infty}\left(\begin{array}{c}n \\ m\end{array}\right)\left(\begin{array}{c}m \\ k\end{array}\right) 2^{n-m+k} M_{1}^{m}(H)\left[M_{1}^{n-m+k}\right.$ $\left.(G) M_{1}(H)+M_{1}^{\alpha-m+k}(G)\left(2+M_{1}(H)\right)\right]$ $+2 \sum_{m=0}^{\infty}\left(\begin{array}{c}n \\ m\end{array}\right) 2^{n+1} e_{G} M_{1}^{m+1}(H)$ (iii) $\begin{aligned} & M^{\alpha}\left(G_{Q} \otimes H\right)=\sum_{m=0}^{\infty} \sum_{k=0}^{\infty}\left(\begin{array}{c}n \\ m\end{array}\right)\left(\begin{array}{c}m \\ k\end{array}\right) M_{1}^{n+k-m}(G) M_{1}^{m}(H) \\ & {\left[M_{1}(H)+\right.}\end{aligned}$

$$
\begin{aligned}
& \left.M_{1}(G)+M_{1}(G) M_{1}(H)\right]+\sum_{m=0}^{\infty} \sum_{i=0}^{\infty}\left(\begin{array}{c}
n \\
m
\end{array}\right)\left(\begin{array}{c}
n \\
i
\end{array}\right)[2+ \\
& \left.2 M_{1}(H)\right] \quad \sum_{u v \in(G)} M_{1}^{m}(H)\left[d_{G}^{n-i}(u) d_{G}^{i}(v)\right]+\sum_{m=0}^{\infty} \\
& \left(\begin{array}{c}
n \\
m
\end{array}\right) \sum_{i=0}^{\infty}\left(\begin{array}{c}
n \\
i
\end{array}\right) \sum_{u v, v w \in E(G)} M_{1}^{m}(H)\left[d_{G}^{n-i}(u) \quad d_{G}^{i}(v)+\right.
\end{aligned}
$$$$
\left.d_{G}^{n-i}(v) d_{G}^{i}(w)\right]\left[1+M_{1}(H)\right]
$$

(iv) $M^{\alpha}\left(G_{T} \otimes H\right)=\sum_{m=0}^{\infty} \sum_{k=0}^{\infty}\left(\begin{array}{c}n \\ m\end{array}\right)\left(\begin{array}{c}m \\ k\end{array}\right) 2_{\infty}^{n-m+k} M_{1}^{m+1}(H)$ $M_{1}^{k-m}(G)\left[M_{1}^{n}(G)+3 M_{1}^{\alpha}(G)\right]+\sum_{m=0}^{\infty}\left(\begin{array}{c}n \\ m\end{array}\right) \sum_{u v \in V(G)} \sum_{i=0}^{\infty}$ 
Table 1: Table for Example 1.

\begin{tabular}{lcccc}
\hline$[m, n, \alpha]$ & $M^{\alpha}\left(P_{n S} \otimes P_{m}\right)$ & $M^{\alpha}\left(P_{n R} \otimes P_{m}\right)$ & $M^{\alpha}\left(P_{n Q} \otimes P_{m}\right)$ & $M^{\alpha}\left(P_{n T} \otimes P_{m}\right)$ \\
\hline$(3,3,3)$ & 1808 & 6414 & 3442 & 8048 \\
$(4,4,4)$ & 37260 & 208104 & 102800 & 258412 \\
$(5,5,5)$ & 434888 & 5505322 & 1845182 & 6915616 \\
$(6,6,6)$ & $5.42 \times 10^{6}$ & 127857836 & 48374140 & 150810676 \\
$(7,7,7)$ & $6.25 \times 10^{7}$ & 2713045998 & 560449256 & 3056832424 \\
$(8,8,8)$ & $6.84 \times 10^{7}$ & $5.39 \times 10^{10}$ & $7.56 \times 10^{10}$ & $5.88 \times 10^{10}$ \\
$(9,9,9)$ & $7.11 \times 10^{10}$ & $1.02 \times 10^{12}$ & $1.01 \times 10^{12}$ & $1.088 \times 10^{12}$ \\
$(10,10,10)$ & $7.34 \times 10^{10}$ & $1.85 \times 10^{13}$ & & $1.94 \times 10^{13}$ \\
\hline
\end{tabular}

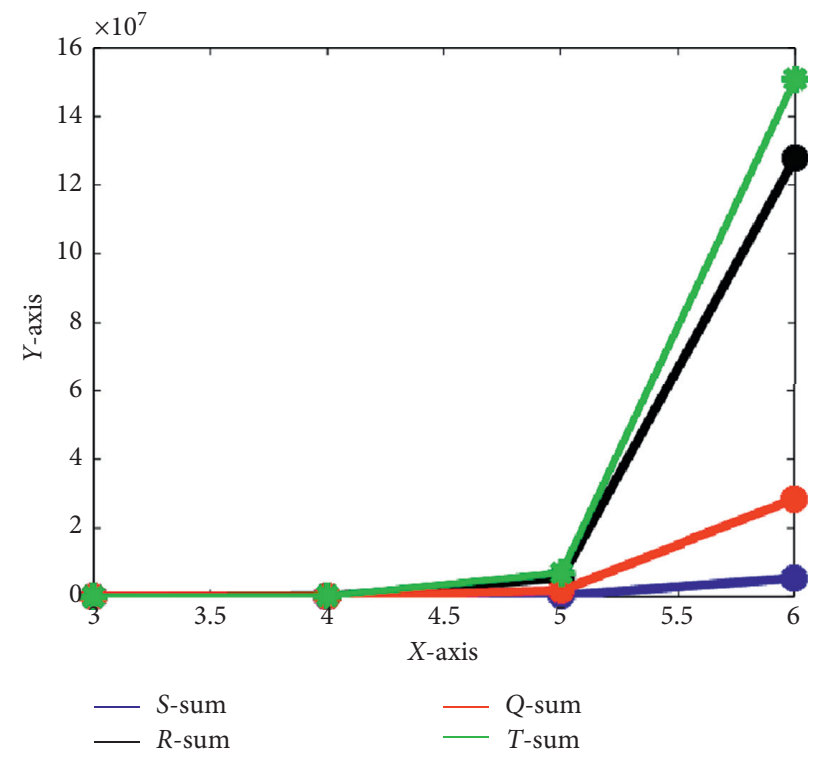

Figure 3: Graphical representations for Table 1.

TABle 2: Table for Example 2.

\begin{tabular}{lcccc}
\hline$[m, n, \alpha]$ & $M^{\alpha}\left(C_{n S} \otimes C_{m}\right)$ & $M^{\alpha}\left(C_{n R} \otimes C_{m}\right)$ & $M^{\alpha}\left(C_{n Q} \otimes C_{m}\right)$ & $M^{\alpha}\left(C_{n T} \otimes C_{m}\right)$ \\
\hline$(3,3,3)$ & 6552 & 26640 & 17568 & 37656 \\
$(4,4,3)$ & 11648 & 47360 & 31232 & 66944 \\
$(5,5,3)$ & 18200 & 74000 & 48800 & 104600 \\
\hline
\end{tabular}

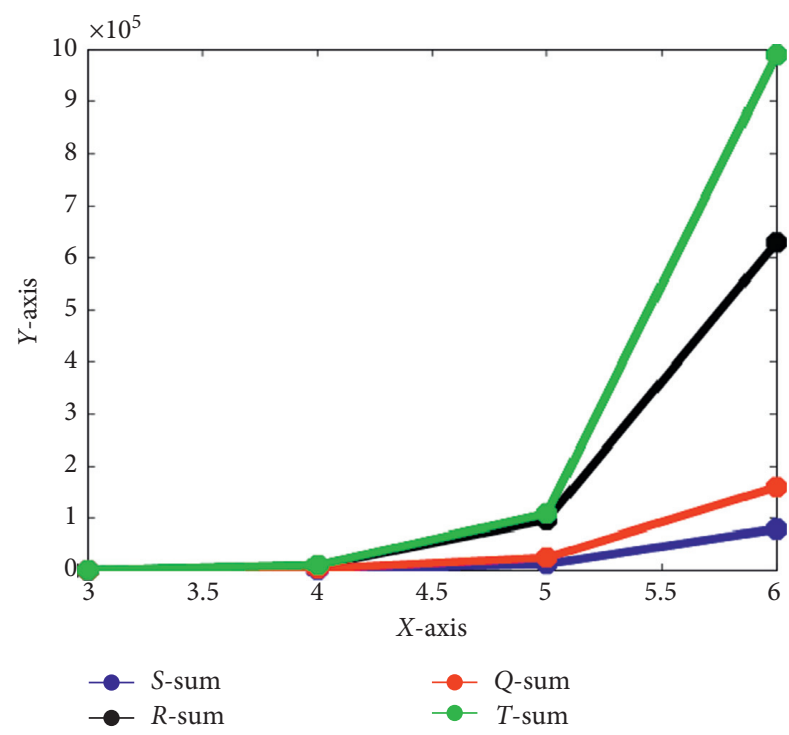

Figure 4: Graphical representations for Table 2. 


$$
\begin{aligned}
& \left(\begin{array}{c}
n \\
i
\end{array}\right)\left[d_{G}^{n-i}(u) d_{G}^{i}(v)\right]\left[3 M_{1}^{m}(H)+3 M_{1}^{m+1}(H)\right] \\
& +\sum_{m=0}^{\infty}\left(\begin{array}{c}
n \\
m
\end{array}\right) \sum_{v w \in V(G)} \sum_{i=0}^{\infty}\left(\begin{array}{c}
n \\
i
\end{array}\right)\left[d_{G}^{n-i}(v) d_{G}^{i}(w)\right]\left[2 M_{1}^{m+1}\right. \\
& \left.(H)+M_{1}^{m}(H)\right] .
\end{aligned}
$$

\section{Discussion and Conclusion}

We conclude our work with applications of (Theorem 1-Theorem 4) in the following examples.

Example 1. For two graphs $P_{n}$ and $P_{m}$ of order $n \geq 3$ and $m \geq 2$, we have

$$
\begin{aligned}
M^{\alpha}\left(P_{n S} \otimes P_{m}\right)= & \sum_{m=0}^{n} \sum_{k=0}^{m} C_{m}^{n} C_{k}^{m}\left[2^{\alpha+k+2}(n-2)(m-2)+5(2)^{\alpha-m+k}(n-2)+5(2)^{m+1}(m-2)+12\right] \\
& +2^{n+1}(n-1) \sum_{m=0}^{n} C_{m}^{n}\left[2^{m}\left(2 m^{2}-5 m+2\right)+4 m-2\right], \\
M^{\alpha}\left(P_{n R} \otimes P_{m}\right)= & \sum_{m=0}^{n} C_{m}^{n}\left[\sum_{k=0}^{m} C_{k}^{m} 2^{n-m+k}\left\{\left(2^{m+1}(m+2)+2\right)\left(2^{\alpha-m+k}(n-3)+8\right)\right\}+2^{n+3}(n-1)\left\{2^{m}(m-1)+1\right\}\right], \\
M^{\alpha}\left(P_{n Q} \otimes P_{m}\right)= & \sum_{m=0}^{n} \sum_{k=0}^{m} C_{m}^{n} C_{k}^{m}\left[2^{\alpha+k+2}(n-2)(m-2)+5(2)^{\alpha-m+k}(n-2)+5(2)(m-2)+12\right] \\
& +\sum_{m=0}^{n} \sum_{i=0}^{n} C_{m}^{n} C_{i}^{n}\left[2^{m}(m-2)+2\right]\left[2^{i}+2^{n}(n-2)+2^{n-i}\right]\left[4(m-1)+2^{i}+2^{n}(n-2)+2^{n-i}+2 m-1\right], \\
M^{\alpha}\left(P_{n T} \otimes P_{m}\right)= & \sum_{m=0}^{n} \sum_{k=0}^{m} C_{m}^{n} C_{k}^{m} 2^{n-m+k}\left[2^{m+1}(m-2)+2\right]\left[2^{k-m}(n-2)+2\right]\left[2^{n}+3(2)^{\alpha}\right]+\sum_{m=0}^{n} \sum_{i=0}^{n} C_{m}^{n} C_{i}^{n} \\
& {\left[\left\{9\left(2^{m}\right)(m-2)+12\right\}\left\{2^{i}+2^{n}(n-2)+2^{n-i}\right\}+\left\{2^{m}(5 m-10)+6\right\}\left\{2^{i}+2^{n}(n-2)+2^{n-i}\right\}^{2}\right] . }
\end{aligned}
$$

Example 2. For two graphs $C_{n}$ and $C_{m}$ of order $n, m \geq 3$, we have

$$
\begin{aligned}
& M^{\alpha}\left(C_{n S} \otimes C_{m}\right)=\sum_{m=0}^{n} \sum_{k=0}^{m} C_{m}^{n} C_{k}^{m}\left[2^{\alpha+k+2}(n m)\right]+\sum_{m=0}^{n} C_{m}^{n}\left[2^{n+m+1} m(n-1)\left(1+2^{m} m\right)\right] \\
& M^{\alpha}\left(C_{n R} \otimes C_{m}\right)=\sum_{m=0}^{n} \sum_{k=0}^{m} C_{m}^{n} C_{k}^{m}\left[2^{2 n+2 k-m+1}(9 m n)\right]+\sum_{m=0}^{n} C_{m}^{n}\left[2^{n+m+3} m(n-1)\right], \\
& M^{\alpha}\left(C_{n Q} \otimes C_{m}\right)=\sum_{m=0}^{n} \sum_{k=0}^{m} C_{m}^{n} C_{k}^{m}\left[2^{\alpha+k+2}(n m)\right]+\sum_{m=0}^{n} \sum_{i=0}^{n} C_{m}^{n} C_{i}^{n}\left[2^{m+n+2} n m(1+2 m)\right] \\
& M^{\alpha}\left(C_{n T} \otimes C_{m}\right)=\sum_{m=0}^{n} \sum_{k=0}^{m} C_{m}^{n} C_{k}^{m}\left[2^{2(n+k+1)-m}(n m)\right]+\sum_{m=0}^{n} \sum_{i=0}^{n} C_{m}^{n} C_{i}^{n}\left[2^{n+m}(14 n m)\right] .
\end{aligned}
$$

(i) In this paper, we computed the exact values of FGZ index of the four $\Phi$-sum graphs $\left(G_{S} \otimes H, G_{R} \otimes H\right.$, $G_{Q} \otimes H$ and $\left.G_{T} \otimes H\right)$ in terms of their factor graphs, where these graphs are obtained under the operation of the strong product.

(ii) It is the extension of Deng et al. [13], Akhter and Imran [14], Liu et al. [15] and Sarala et al. [16] works. (iii) The FGZ index of the $\Phi$-sum graphs obtained from the paths and cycles are also computed as applications of the obtained results.

(iv) Table 1 and Figure 3 present that FGZ index of $P_{n T}$ $\otimes P_{m}$ is dominant having ordering $M^{\alpha}\left(P_{n T} \otimes P_{m}\right)$ $\geq M^{\alpha}\left(P_{n R} \otimes P_{m}\right) \geq M^{\alpha}\left(P_{n Q} \otimes P_{m}\right) \geq M^{\alpha}\left(P_{n S} \otimes P_{m}\right)$.

(v) Table 2 and Figure 4 also presents that FGZ index of $C_{n T} \otimes C_{m} \quad$ is dominant having ordering 


$$
\begin{aligned}
& M^{\alpha}\left(C_{n T} \otimes C_{m}\right) \geq M^{\alpha} \quad\left(C_{n R} \otimes C_{m}\right) \geq M^{\alpha}\left(C_{n Q} \otimes C_{m}\right) \\
& \geq M^{\alpha}\left(C_{n S} \otimes C_{m}\right) .
\end{aligned}
$$

\section{Data Availability}

All data are included within this paper. However, the reader may contact the corresponding author for more details of the data.

\section{Disclosure}

The authors have no conflicts of interest.

\section{Conflicts of Interest}

The authors declare that they have no conflicts of interest.

\section{Acknowledgments}

The author is deeply thankful to the reviewers for their valuable suggestions to improve the quality of this manuscript. This work was supported by the Humanities and Social Science Project of Anhui Provincial Education Department. Research on social crisis management in Anhui cities under the background of big data (Subject No: SK2016A0233).

\section{References}

[1] Z. Ahmad, M. Naseem, M. Naseem, M. K. Jamil, M. F. Nadeem, and S. Wang, "Eccentric connectivity indices of titania nanotubes $\mathrm{TiO}_{2}[m ; n]$," Eurasian Chemical Communications, vol. 2, no. 6, pp. 712-721, 2020.

[2] S. Imran, M. Siddiqui, M. Imran, and M. Nadeem, "Computing topological indices and polynomials for line graphs," Mathematics, vol. 6, no. 8, p. 137, 2018.

[3] Z. Zhang, Z. S. Mufti, M. F. Nadeem, Z. Ahmad, M. K. Siddiqui, and M. R. Farahani, "Computing topological indices for para-line graphs of anthracene," Open Chemistry, vol. 17, no. 1, pp. 955-962, 2019.

[4] O. M. Minailiuc and M. V. Diudea, "TI-MTD model applications in molecular design," QSPR/QSAR Studies by Molecular Descriptors, vol. 32, no. 27, pp. 363-388, 2001.

[5] I. Gutman and N. Trinajstić, Graph Theory and Molecular Orbitals, pp. 49-93, New Concepts Springer, Berlin, Germany, 1973.

[6] H. Wiener, "Structural determination of paraffin boiling points," Journal of the American Chemical Society, vol. 69, no. 1, pp. 17-20, 1947.

[7] I. Gutman and N. Trinajstić, "Graph theory and molecular orbitals. total $\varphi$-electron energy of alternant hydrocarbons," Chemical Physics Letters, vol. 17, no. 4, pp. 535-538, 1972.

[8] L. B. Kier and L. H. Hall, "Structure-activity studies on hallucinogenic amphetamines using molecular connectivity," Journal of Medicinal Chemistry, vol. 20, no. 12, pp. 1631-1636, 1977.

[9] X. Li and J. Zheng, "A unified approach to the extremal trees for different indices," MATCH, Communications in Mathematical and in Computer Chemistry, vol. 54, no. 1, pp. 195-208, 2005.

[10] N. Akgunes, K. C. Das, A. S. Cevik, and I. N. Cangul, "Some properties on the lexicographic product of graphs obtained by monogenic semigroups," Journal of Inequalities and Applications, vol. 238, no. 1, pp. 1-9, 2013.
[11] W. Yan, B.-Y. Yang, and Y.-N. Yeh, "The behavior of Wiener indices and polynomials of graphs under five graph decorations," Applied Mathematics Letters, vol. 20, no. 3, pp. 290-295, 2007.

[12] M. Eliasi and B. Taeri, "Four new sums of graphs and their Wiener indices," Discrete Applied Mathematics, vol. 157, no. 4, pp. 794-803, 2009.

[13] H. Deng, D. Sarala, S. K. Ayyaswamy, and S. Balachandran, "The Zagreb indices of four operations on graphs," Applied Mathematics and Computation, vol. 275, pp. 422-431, 2016.

[14] S. Akhter and M. Imran, "Computing the forgotten topological index of four operations on graphs," AKCE International Journal of Graphs and Combinatorics, vol. 14, no. 1, pp. 70-79, 2017.

[15] J.-B. Liu, S. Javed, M. Javaid, and K. Shabbir, "Computing first general Zagreb index of operations on graphs," IEEE Access, vol. 7, pp. 47494-47502, 2019.

[16] D. Sarala, H. Deng, C. Natarajan, and S. K. Ayyaswamy, "F index of graphs based on four new operations related to the strong product," AKCE International Journal of Graphs and Combinatorics, vol. 17, no. 1, pp. 25-37, 2020.

[17] H. M. Awais, M. Javaid, and A. Akbar, "First general Zagreb index of generalized F-sum graphs," Discrete Dynamics in Nature and Society, vol. 2020, Article ID 2954975, 16 pages, 2020.

[18] H. M. Awais, M. Jamal, and M. Javaid, “Topological properties of metal-organic frameworks," Main Group Metal Chemistry, vol. 43, no. 1, pp. 67-76, 2020.

[19] H. M. Awais, M. Javaid, and M. Jamal, "Forgotten index of generalized F-sum graphs," Journal of Prime Research in Mathematics, vol. 15, pp. 115-128, 2019.

[20] H. M. Awais, M. Javaid, and A. Raheem, "Hyper-Zagreb index of graphs based on generalized subdivision related operations," Punjab University Journal of Mathematics, vol. 52, no. 5, pp. 89-103, 2019.

[21] Y.-M. Chu, S. Javed, M. Javaid, and M. Kamran Siddiqui, "On bounds for topological descriptors of $\varphi$-sum graphs," Journal of Taibah University for Science, vol. 14, no. 1, pp. 1288-1301, 2020.

[22] G. Hong, Z. Gu, M. Javaid, H. M. Awais, and M. K. Siddiqui, "Degree-based topological invariants of metal-organic networks," IEEE Access, vol. 8, pp. 68288-68300, 2020.

[23] M. Javaid, S. Javed, A. M. Alanazi, and M. R. Alotaibi, "Computing analysis of Zagreb indices for generalized sum graphs under strong product," Journal of Chemistry, vol. 2021, Article ID 6663624, 20 pages, 2021.

[24] J. B. Liu, Z. Raza, and M. Javaid, "Zagreb connection numbers for cellular neural networks," Discrete Dynamics in Nature and Society, vol. 2020, Article ID 8038304, 8 pages, 2020.

[25] J.-B. Liu, J. Zhao, S. Wang, M. Javaid, and J. Cao, "On the topological properties of the certain neural networks," Journal of Artificial Intelligence and Soft Computing Research, vol. 8, no. 4, pp. 257-268, 2018.

[26] U. Ahmad and S. Hameed, "Study of topological indices in a class of benzenoid graphs," Computational Journal of Combinatorial Mathematics, vol. 1, pp. 19-30, 2020.

[27] X. Zhang, H. M. Awais, M. Javaid, and M. K. Siddiqui, "Multiplicative Zagreb indices of molecular graphs," Journal of Chemistry, vol. 2019, Article ID 5294198, 19 pages, 2019.

[28] I. Gutman, B. Ruščić, N. Trinajstić, and C. F. Wilcox, "Graph theory and molecular orbitals. XII. acyclic polyenes," The Journal of Chemical Physics, vol. 62, no. 9, pp. 3399-3405, 1975. 
[29] J. B. Liu, M. Javaid, and H. M. Awais, "Computing Zagreb indices of the subdivision-related generalized operations of graphs," IEEE Access, vol. 7, pp. 105479-105488, 2019.

[30] K. C. Das, N. Akgunes, M. Togan, A. Yurttas, I. N. Cangul, and A. S. Cevik, "On the first Zagreb index and multiplicative Zagreb coindices of graphs," Analele Universitatii "Ovidius" Constanta-Seria Matematica, vol. 24, no. 1, pp. 153-176, 2016.

[31] X. Li, M. Ahmad, M. Javaid, M. Saeed, and J. B. Liu, "Bounds on general randic index for F-sum graphs," Journal of Mathematics, vol. 2020, Article ID 9129365, 17 pages, 2020. 